\title{
Intrinsic Kinetic Modeling of Hydrogen Production by Photocatalytic Water Splitting Using Cadmium Zinc Sulfide Catalyst
}

\author{
Hyacinth Mae G. Tambago and Rizalinda L. de Leon
}

\begin{abstract}
Photocatalytic water splitting is an alternative method for hydrogen production. Cadmium zinc sulfide is a photocatalyst active under visible light that is effective for hydrogen generation in the presence of sulfide and sulfite as sacrificial reagents. Design of photocatalytic reactors for such a system requires a kinetic model of the photocatalytic reaction. In this study, the effect of catalyst loading, sulfide and sulfite concentration, and incident light intensity on the rate of hydrogen production using a cadmium zinc sulfide catalyst suspended in an externally irradiated batch reactor was investigated. The variation of the local volumetric rate of photon absorption (LVRPA) in the batch reactor was taken into account by modeling the reactor radiation field using the two-flux theory of Kubelka and Munk. A kinetic model reflecting the influence of sacrificial reagent concentrations and the LVRPA was developed and fit to the experimental data. The model parameters obtained are independent of irradiation form and reactor geometry and may thus be used for the design and scale-up of photocatalytic reactors.
\end{abstract}

Index Terms-Cadmium zinc sulfide, intrinsic kinetic modeling, photocatalytic hydrogen production, water splitting.

\section{INTRODUCTION}

The environmental problems caused by the current consumption of fossil fuels as a major energy source, which are particularly being associated with global warming, and its eventual depletion, have led the move towards clean and renewable energy sources. Hydrogen is a promising energy carrier as it undergoes clean combustion. At present, however, majority of the world's hydrogen is generated from steam reforming of natural gas, a fossil fuel, which also results in carbon dioxide emissions [1].

Alternative methods of hydrogen production include water splitting, which is the dissociation of water into hydrogen and oxygen gas, as shown in (1), using light in the presence of a photocatalyst. Since the discovery of water splitting on a photoelectrochemical cell comprised of $\mathrm{a} \mathrm{TiO}_{2}$ photo-anode and a Pt photo-cathode under ultraviolet (UV) irradiation by Honda and Fujishima in 1972, a lot of research on photocatalytic water splitting has been conducted.

Manuscript received August 10, 2014; revised November 7, 2014. This work was supported in part by the University of the Philippines Engineering Research and Development Foundation, Inc. (UPERDFI) Research and Development Fellowship and Incentive Award and the Engineering Research and Development for Technology (ERDT) under the Republic of the Philippines Department of Science and Technology (DOST).

H. M. G. Tambago and R. L. de Leon are with the Department of Chemical Engineering, University of the Philippines, Diliman, 1108 Quezon City, Philippines (e-mail: hyacinth.tambago@coe.upd.edu.ph, rldeleon@upd.edu.ph).

$$
\mathrm{H}_{2} \mathrm{O} \rightarrow \mathrm{H}_{2}+\frac{1}{2} \mathrm{O}_{2} \quad \Delta G^{\circ}=238 \mathrm{~kJ} / \mathrm{mol}
$$

Simultaneous generation of hydrogen and oxygen on a single photocatalyst particle under visible light irradiation is difficult to achieve since the photocatalyst must be able to satisfy band position requirements and its band gap must not be too large. In addition, fast electron-hole recombination and backward reaction of hydrogen and oxygen to water reduce the efficiency of overall water splitting. The generation of a mixture of hydrogen and oxygen also calls for a separation process that still has to be developed in order to obtain pure hydrogen.

In order to enhance photocatalytic hydrogen generation, sacrificial reagents such as alcohols and sulfide ions are added to the aqueous suspension of the photocatalyst. Also called hole scavengers, these reducing agents enrich electrons by being irreversibly oxidized instead of water by photo-generated holes. Recombination of electron and hole is minimized and thus hydrogen production is enhanced. However, when the sacrificial reagent becomes used up, hydrogen production is inhibited. This kind of photocatalytic hydrogen-generating system thus calls for a continual addition of hole scavengers and is meaningful if industrial polluting by-products, biomass, and compounds abundant in nature are used as reducing reagents [2].

Metal oxide photocatalysts such as $\mathrm{TiO}_{2}, \mathrm{SrTiO}_{3}$, and other perovskite titanium and tantalum compounds are commonly used for water splitting due to their stability during the photoreaction in water. These photocatalysts, however, have too wide band gaps that make them responsive only to UV light, which comprises only about $6 \%$ of solar radiation. Modifications such as doping with cations or anions and loading noble metal co-catalysts are conducted to make these catalysts active under visible light and more effective for hydrogen generation.

Cadmium sulfide (CdS) is a well-known metal sulfide catalyst that is active under visible light and that satisfies the band position requirements for water splitting. However, this catalyst undergoes photocorrosion in which the sulfur anion of the catalyst becomes oxidized by the photogenerated holes to $\mathrm{S}$ or $\mathrm{S}_{2}{ }^{2-}$ as water is reduced to hydrogen. Sulfur may deposit on the catalyst surface and $\mathrm{S}_{2}{ }^{2-}$ competes with the catalyst for absorption of photons in the visible light region. In order to prevent these, sulfide $\left(\mathrm{S}^{2-}\right)$ and sulfite $\left(\mathrm{SO}_{3}{ }^{2-}\right)$ ions are added to the catalyst suspension as sacrificial reagents. The sulfide species becomes oxidized to $\mathrm{S}$ or $\mathrm{S}_{2}{ }^{2-}$ instead of the sulfide ion in the catalyst and sulfite reacts with $\mathrm{S}$ and $\mathrm{S}_{2}{ }^{2-}$ to form thiosulfate $\left(\mathrm{S}_{2} \mathrm{O}_{3}{ }^{2-}\right)$, which is a colorless species. Modifications on the CdS catalyst are performed to improve its efficiency and stability such as loading Pt and other noble 
metals as co-catalyst or incorporating $\mathrm{ZnS}$, another metal sulfide catalyst that is used for hydrogen production but only active under UV light, into CdS to form a solid solution of $\mathrm{Cd}_{x} \mathrm{Zn}_{1-x} \mathrm{~S}$. Synthesized $\mathrm{Cd}_{x} \mathrm{Zn}_{1-x} \mathrm{~S}$ without loaded noble metals has been found to be effective for hydrogen production [3], which is beneficial for cost-effective photocatalyst production without addition of expensive metal co-catalysts [4].

A practical application on the use of sulfide and sulfite as sacrificial reagents in metal sulfide catalytic systems is the utilization of waste streams containing these species. Hydrogenation and flue-gas desulfurization processes generate hydrogen sulfide and sulfur dioxide as by-products, which exist in sulfide and sulfite forms, respectively, in alkaline solutions. Use of these waste streams for hydrogen production is interesting since it simultaneously accomplishes both waste treatment and renewable energy generation [2]. For the design of reactors for this photocatalytic system, a kinetic model that shows the influence of the system parameters such as the sacrificial reagent concentration and light intensity is required.

This study attempts to address the need of a complete photoreactor design for hydrogen production using a $\mathrm{Cd}_{x} \mathrm{Zn}_{1-x} \mathrm{~S}$ photocatalyst with sulfide and sulfite for an intrinsic kinetic model that will be coupled with mass transfer, hydrodynamics, and radiation transfer models. The model should reflect the influence of sulfide and sulfite concentrations and photon absorption in the form of the local volumetric rate of photon absorption (LVRPA). The LVRPA corresponds to the amount of photons incident on a region of the reactor that is absorbed by the photocatalyst. Due to attenuation of light caused by the absorbing species such as the photocatalyst in suspension, the radiation field and the LVRPA is not uniform throughout the reactor. The LVRPA and its variation in the reactor are dependent on several system properties such as the intensity and spectral irradiance of the light source, the geometrical configuration of the reactor, the amount of the catalyst, and the absorption and scattering properties of the catalyst and other species in the system. In order for the kinetic model to be used for photoreactor design and scale-up, the model parameters, which are derived from kinetic studies carried out in an irradiated batch reactor, must be independent of the experimental configuration; hence, the radiation field in the photoreactor used in the kinetic studies must be modeled in order to consider the variation of the LVRPA in the photoreactor. This was performed in [5] in order to determine the intrinsic kinetic model of photocatalytic oxidation of cyanide in water using a $\mathrm{TiO}_{2}$ catalyst under UV irradiation.

This study aims to develop a model for the intrinsic kinetics of photocatalytic hydrogen production via water splitting on a synthesized $\mathrm{Cd}_{0.4} \mathrm{Zn}_{0.6} \mathrm{~S}$ photocatalyst in the presence of sulfide and sulfite ions as sacrificial reagents. Radiation absorption will be considered for estimation of parameters that will be independent of experimental conditions.

\section{EXPERIMENTAL}

\section{A. Photocatalyst}

The $\mathrm{Cd}_{0.4} \mathrm{Zn}_{0.6} \mathrm{~S}$ photocatalyst used in this study, for which hydrogen production was found to be highest among three values of $x$ in $\mathrm{Cd}_{x} \mathrm{Zn}_{1-x} \mathrm{~S}$ considered, was synthesized via the co-precipitation method [6]. Cadmium acetate dihydrate, $\mathrm{Cd}\left(\mathrm{CH}_{3} \mathrm{COO}\right)_{2} \cdot 2 \mathrm{H}_{2} \mathrm{O}$ and zinc acetate dihydrate, $\mathrm{Zn}\left(\mathrm{CH}_{3} \mathrm{COO}\right)_{2} \cdot 2 \mathrm{H}_{2} \mathrm{O}$ were dissolved in $100 \mathrm{~mL}$ of deionized water. The solution was constantly stirred and added drop wise with $0.1 \mathrm{M}$ sodium sulfide, $\mathrm{Na}_{2} \mathrm{~S} \cdot \mathrm{H}_{2} \mathrm{O}$. The amounts of the reagents were such that the $\mathrm{Cd}: \mathrm{Zn}: \mathrm{S}$ atomic ratio is 0.4:0.6:1. The formed precipitate was separated from the solution by vacuum filtration, washed with deionized water, and dried at $348 \mathrm{~K}$ for eight (8) hours.

\section{B. Photocatalytic Reactor}

Photocatalytic reactions were carried out in a Pyrex cylindrical vessel with an O-ring joint seal. The catalyst suspension is held in the portion of the vessel below the joint having an inner diameter of $5 \mathrm{~cm}$ and height of $5 \mathrm{~cm}$. The joints make up the headspace with a volume of $126 \mathrm{~cm}^{3}$. On the top of the reactor is a sample port with a polytetrafluoroethylene (PTFE) septum for extraction of gas samples from the headspace.

The reactor is irradiated through one side with a $400-\mathrm{W}$ tubular halogen lamp (Osram Haloline Eco SST). Between the lamp and the reactor is a UV cut-off filter. The surface of this side of the vessel facing the lamp was made to have a ground glass texture to realize the assumption of a diffuse incoming radiation used in modeling the radiation field inside the reactor. The irradiance of the lamp was measured using a DayStar DS 05 solar meter and the lamp spectral irradiance was determined by an Ocean Optics HR2000+ high-resolution spectrometer. Temperature in the reactor was maintained at $25 \pm 1^{\circ} \mathrm{C}$ by a Peltier cell cooling system.

\section{Photocatalytic Reaction Runs}

The photocatalyst suspension is composed of the $\mathrm{Cd}_{0.4} \mathrm{Zn}_{0.6} \mathrm{~S}$ photocatalyst particles in an aqueous solution of sodium sulfide $\left(\mathrm{Na}_{2} \mathrm{~S}\right)$ and sodium sulfite $\left(\mathrm{Na}_{2} \mathrm{SO}_{3}\right)$ prepared by dissolving prescribed amounts of sodium sulfide hydrate flakes and anhydrous $\mathrm{Na}_{2} \mathrm{SO}_{3}$ in $100 \mathrm{~mL}$ of deionized water. Hydroxide concentration in the catalyst suspension was determined from $\mathrm{pH}$ measurements using a $\mathrm{pH}$ meter (CyberScan $\mathrm{pH}$ 110). The photocatalytic reaction runs were conducted at varying catalyst loading, sulfide concentration, sulfite concentration, and incident light intensity, as shown in Table I. Variation of the incident light intensity was done by varying the distance of the lamp from the reactor. The experimental error was estimated to be the standard deviation of three replicates of Run 3.

Prior to each run, the reactor suspension was purged with nitrogen gas for 15 minutes to remove oxygen. Initial rates of photocatalytic hydrogen production were determined by taking hourly measurements of the amount of hydrogen in the reactor headspace for four (4) hours. A 1-mL gas sample is extracted from the headspace through the PTFE septum using a 2.5-mL gas-tight syringe (Hamilton HD-type) and injected onto a gas chromatograph (GC) with a thermal conductivity detector (Shimadzu GC 2014) using a molecular sieve column (Supelco Mol Sieve 5A) and nitrogen as the carrier gas. A calibration curve relating the chromatogram peak area units to the amount of hydrogen (in micromoles) was created by injecting different volumes of a $1.2 \% \quad \mathrm{H}_{2}-\mathrm{in}-\mathrm{N}_{2}$ gas standard onto the GC. 
TABLE I: PHOTOCATALYTIC REACTION RUNS

\begin{tabular}{|c|c|c|c|c|c|}
\hline Run & $\begin{array}{c}\text { Mass of } \\
\text { catalyst, } \mathrm{g} \\
\text { in } 100 \mathrm{~mL} \\
\text { solution }\end{array}$ & $\begin{array}{c}{\left[\mathrm{Na}_{2} \mathrm{~S}\right]} \\
\mathrm{M}\end{array}$ & $\begin{array}{c}{\left[\mathrm{Na}_{2} \mathrm{SO}_{3}\right],} \\
\mathrm{M}\end{array}$ & $\begin{array}{c}{\left[\mathrm{OH}^{-}\right],} \\
\mathrm{M}\end{array}$ & $\begin{array}{c}\text { Incident light } \\
\text { intensity, } \\
\mathrm{mW} / \mathrm{cm}^{2}\end{array}$ \\
\hline 1 & 0.025 & 0.1 & 0.1 & 0.92 & 54.95 \\
\hline 2 & 0.075 & 0.1 & 0.1 & 0.92 & 54.95 \\
\hline 3 & 0.1 & 0.1 & 0.1 & 0.92 & 54.95 \\
\hline 4 & 0.2 & 0.1 & 0.1 & 0.92 & 54.95 \\
\hline 5 & 0.1 & 0.005 & 0.1 & 0.07 & 54.95 \\
\hline 6 & 0.1 & 0.01 & 0.1 & 0.14 & 54.95 \\
\hline 7 & 0.1 & 0.025 & 0.1 & 0.32 & 54.95 \\
\hline 8 & 0.1 & 0.05 & 0.1 & 0.61 & 54.95 \\
\hline 9 & 0.1 & 0.3 & 0.1 & 1.28 & 54.95 \\
\hline 10 & 0.1 & 0.1 & 0.01 & 0.65 & 54.95 \\
\hline 11 & 0.1 & 0.1 & 0.025 & 0.66 & 54.95 \\
\hline 12 & 0.1 & 0.1 & 0.05 & 0.73 & 54.95 \\
\hline 13 & 0.1 & 0.1 & 0.3 & 0.99 & 54.95 \\
\hline 14 & 0.1 & 0.1 & 0.1 & 0.92 & 69.00 \\
\hline 15 & 0.1 & 0.1 & 0.1 & 0.92 & 42.91 \\
\hline 16 & 0.1 & 0.1 & 0.1 & 0.92 & 34.84 \\
\hline
\end{tabular}

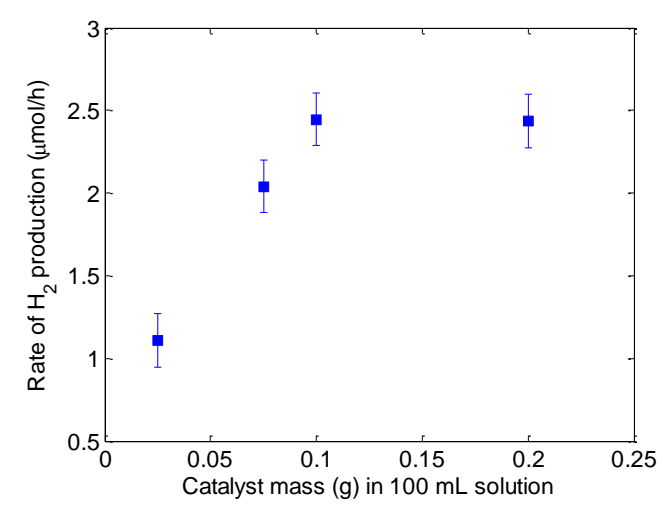

Fig. 1. Initial rates of hydrogen production at various amounts of catalyst (100 mL solution with $0.1 \mathrm{M} \mathrm{Na} 2 \mathrm{~S}, 0.1 \mathrm{M} \mathrm{Na}_{2} \mathrm{SO}_{3}, 54.95 \mathrm{~mW} / \mathrm{cm}^{2}$ incident irradiance).

\section{EXPERIMENTAL RATES OF HydRogen PRODUCTION}

Figs. 1 to 4 show the experimental rates of hydrogen production at varying operating parameters. It can be observed from Fig. 1 that increasing the catalyst loading results in higher rates of hydrogen production due to increased rates of photon absorption; increasing the concentration of catalyst particles further, however, does not improve the hydrogen generation rate as much due to the shadowing effect of the catalyst particles. The high photon absorption rate by catalyst particles near the irradiated surface of the reactor prevents penetration of light further into the reactor, lowering the total photon absorption that would have supposedly increased due to the higher concentration of light-absorbing particles. As shown in Fig. 2, increasing sulfide concentrations promotes hydrogen evolution due to higher rates of hole consumption, preventing the recombination of electrons and holes. Too high sulfide concentrations, however, decreases the rate of hydrogen production due to the reduction of adsorption sites needed for hydrogen generation and the absorption of light in the visible region by trace polysulfides and sulfide oxidation products, competing with the photocatalyst. This results in the bell shape of the plot of the gas production rate vs. sulfide concentration that is typical for reactions following this type of mechanism, as was also found in [7], [8] for a $\mathrm{CdS}$ photocatalyst. Sulfite ions are needed to convert the sulfide oxidation products to thiosulfate, which is colorless. However, as can be seen in Fig. 3, increasing sulfite ions decreases the hydrogen generation rate due to competitive adsorption of sulfite on the catalyst sites with sulfide [7], [8]. As the incident light intensity is increased, the rate of hydrogen generation becomes higher as shown in Fig. 4 due to the photogeneration of more electrons and holes.

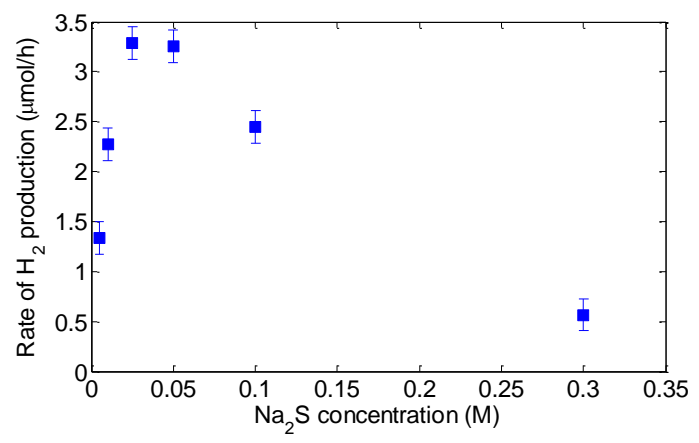

Fig. 2. Initial rates of hydrogen production at various $\mathrm{Na}_{2} \mathrm{~S}$ concentrations ( $0.1 \mathrm{~g}$ catalyst in $100 \mathrm{~mL}$ solution with $0.1 \mathrm{M} \mathrm{Na}_{2} \mathrm{SO}_{3}, 54.95 \mathrm{~mW} / \mathrm{cm}^{2}$ incident irradiance).

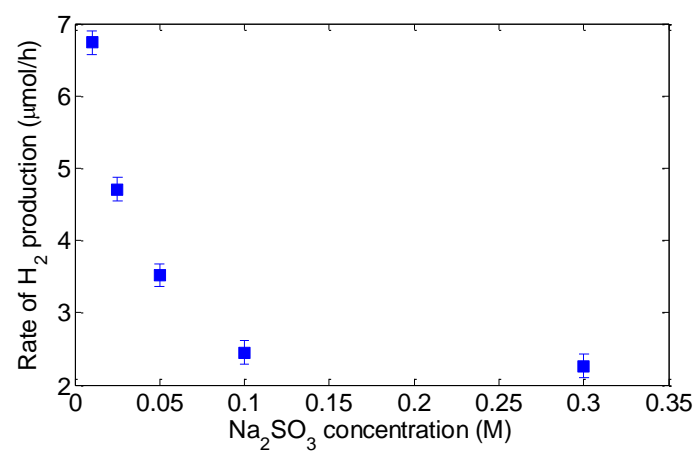

Fig. 3. Initial rates of hydrogen production at various $\mathrm{Na}_{2} \mathrm{SO}_{3}$ concentrations $\left(0.1 \mathrm{~g}\right.$ catalyst in $100 \mathrm{~mL}$ solution with $0.1 \mathrm{M} \mathrm{Na}_{2} \mathrm{~S}, 54.95 \mathrm{~mW} / \mathrm{cm}^{2}$ incident

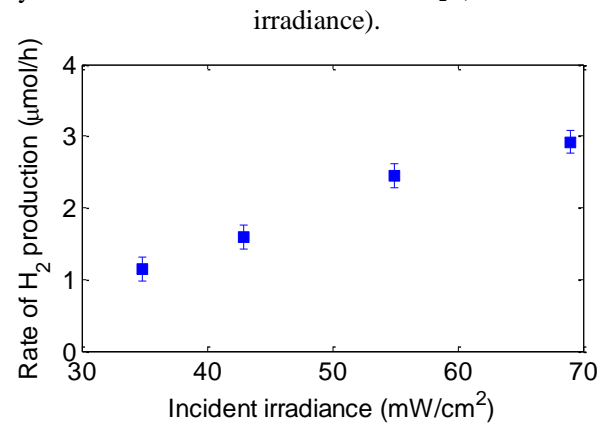

Fig. 4. Initial rates of hydrogen production at various incident irradiances $\left(0.1 \mathrm{~g}\right.$ catalyst in $100 \mathrm{~mL}$ solution with $\left.0.1 \mathrm{M} \mathrm{Na}_{2} \mathrm{~S}, 0.1 \mathrm{M} \mathrm{Na}_{2} \mathrm{SO}_{3}\right)$.

\section{RADIATION FIELD MODELING AND DETERMINATION OF THE LVRPA}

The photocatalytic reactor is not homogeneously irradiated and thus the radiation distribution must be considered in determining intrinsic kinetic parameters independent of the irradiation form, lamp-reactor configuration, and photoreactor geometry. The intensity profile is determined by solving the radiative transfer equation (RTE) applied to the photoreactor. The RTE at steady-state condition and with the assumption of negligible radiation emission is given by the 
following energy balance along a path $d s$ (e.g., in $\mathrm{cm})$ [9]:

$$
\begin{aligned}
\frac{d I_{\lambda, \underline{\Omega}}(\underline{x})}{d s}=- & \kappa_{\lambda} I_{\lambda, \underline{\underline{\Omega}}}(\underline{x})-\sigma_{\lambda} I_{\lambda, \underline{\underline{\Omega}}}(\underline{x}) \\
& +\frac{\sigma_{\lambda}}{4 \pi} \int_{\Omega^{\prime}=4 \pi} p_{\lambda}\left(\underline{\Omega^{\prime}} \cdot \underline{\Omega}\right) I_{\lambda, \underline{\Omega}^{\prime}}(\underline{x}) d \Omega^{\prime}
\end{aligned}
$$

where $I_{\lambda, \Omega}$ (e.g., in $\mathrm{mW} / \mathrm{cm}^{2} \mathrm{sr}$ ) is the wavelength- and direction-dependent intensity, $\underline{x}$ (e.g., in $\mathrm{cm}$ ) is the position vector in a three-dimensional space, $\underline{\Omega}$ is the unit vector in the direction of radiation propagation, $\Omega$ (sr) is the solid angle of radiation propagation about the direction $\underline{\Omega}, \kappa_{\lambda}$ (e.g., in $\mathrm{cm}^{-1}$ ) is the wavelength-dependent absorption coefficient, $\sigma_{\lambda}$ (e.g., in $\mathrm{cm}^{-1}$ ) is the wavelength-dependent scattering coefficient, and $p_{\lambda}\left(\underline{\Omega}^{\prime} \cdot \underline{\Omega}\right)$ is the scattering phase function, which describes the angular distribution of scattered radiation. The left-hand term of (2) represents the change of energy along the direction $\underline{\Omega}$. The first term of the right side of the equation represents the energy loss due to absorption of light along the direction $\underline{\Omega}$, the second is the energy loss due to scattering-out of light from the direction $\underline{\Omega}$, and the third term is the gain of energy along the direction $\underline{\Omega}$ due to the scattering-in of light from all directions $\Omega^{\prime}$ in space.

Application of the complete form of the RTE shown in (2) results in a set of simultaneous integro-differential equations, the solution for which requires a rigorous numerical procedure. One simplification to the RTE is given by the two-flux theory of Kubelka and Munk, which originated from the Schuster equation for isotropic scattering. The Schuster equation considers the radiation field to be consisting of two oppositely directed radiation fluxes, $I_{\lambda}$ (e.g., in J s $\mathrm{m}^{-2} \mathrm{~nm}^{-1}$ ) in the positive or incident radiation propagation direction and $J_{\lambda}$ (e.g., in $\mathrm{J} \mathrm{s}^{-1} \mathrm{~m}^{-2} \mathrm{~nm}^{-1}$ ), which arises from scattering, in the negative propagation direction. The propagation direction is perpendicular to an irradiated layer of thickness $d$ (defined along the $x$-axis), as shown in Fig. 5. The width and length (along the $y z$-plane) of this layer are assumed to be larger than $d$ such that edge effects can be ignored.

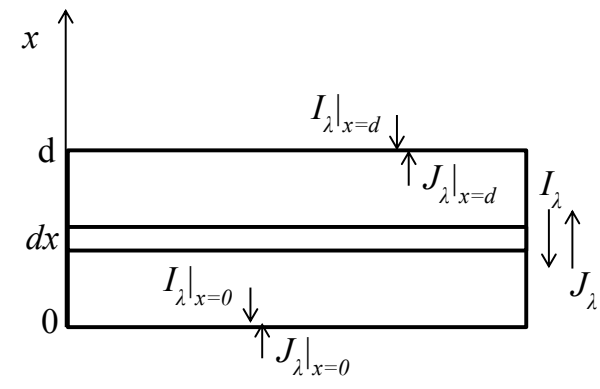

Fig. 5. Irradiated layer modeled by the Schuster equation for isotropic scattering [10].

The theory assumes isotropic distribution of scattering, diffuse incident irradiation, random distribution of the particles in the layer, and that the particles are much smaller than the layer thickness. Under these assumptions, radiation balance within the layer results in the following simultaneous differential equations for $I_{\lambda}$ and $J_{\lambda}[10]$ :

$$
-d I_{\lambda} / d x=-\left(K_{\lambda}+S_{\lambda}\right) I_{\lambda}+S_{\lambda} J_{\lambda}
$$

$$
d J_{\lambda} / d x=-\left(K_{\lambda}+S_{\lambda}\right) J_{\lambda}+S_{\lambda} I_{\lambda}
$$

The parameters $K_{\lambda}$ and $S_{\lambda}$ are defined in terms of the absorption and scattering coefficients of the layer as follows:

$$
K_{\lambda} \equiv 2 \kappa_{\lambda} \quad S_{\lambda} \equiv 2 \sigma_{\lambda} .
$$

The wavelength-dependent specific absorption and scattering coefficients of $\mathrm{Cd}_{0.4} \mathrm{Zn}_{0.6} \mathrm{~S}$ suspensions in water were determined from diffuse transmittance spectrophotometric measurements by solving the RTE applied to the spectrophotometric cells. These optical properties, along with the absorption coefficient of the sacrificial reagent solution and the lamp spectral irradiance, were used to determine the intensity and the LVRPA distributions in the photoreactor.

The modeling procedure for determining the irradiance and LVRPA profile in the reactor is based on a previous work that applied the Kubelka and Munk model to a cylindrical photoreactor externally irradiated by a radially emitting light source [11]. Their procedure, in which the backward flux $J_{\lambda}$ was neglected, was modified in this work in order to take into account both directional fluxes. The photoreactor was discretized into segments with length $\Delta r$ and angular width $\Delta \theta$, for which the fluxes $I_{\lambda}$ and $J_{\lambda}$ entering and leaving the layer were computed. A non-reflecting background was assumed, in which $J_{\lambda}$ at the reactor wall far from the lamp is zero, and the measured incident irradiance at the irradiated wall of the reactor was the other boundary condition used. In addition, the assumption of diffuse incoming radiation used in the radiation field model was realized by the ground glass texture of the reactor wall facing the lamp.

The total incident irradiance $G_{\lambda}$ (e.g., in $J \mathrm{~s}^{-1} \mathrm{~m}^{-2} \mathrm{~nm}^{-1}$ ) at one wavelength $\lambda$ on a particular segment $i$ is the sum of $I_{\lambda}$ and $J_{\lambda}$ entering that segment.

$$
\left.G_{\lambda}\right|_{i}=\left.I_{\lambda}\right|_{i}+\left.J_{\lambda}\right|_{i}
$$

The wavelength-dependent local volumetric rate of energy absorption (LVREA, e.g., in $\mathrm{J} \mathrm{s}^{-1} \mathrm{~m}^{-3} \mathrm{~nm}^{-1}$ ) by the photocatalyst particles at each segment is computed as:

$$
\left.\left.\operatorname{LVREA}\right|_{\lambda}\right|_{i}=\left.K_{\lambda}{ }^{*} C_{c a t} G_{\lambda}\right|_{i}
$$

where $C_{\text {cat }}$ (e.g., in $\mathrm{g} \mathrm{cm}^{-3}$ ) is the catalyst concentration and $K_{\lambda}^{*}$ (e.g., in $\mathrm{cm}^{2} \mathrm{~g}^{-1}$ ) is the $K_{\lambda}$ parameter per unit catalyst concentration.

The LVRPA (e.g., in photons $\mathrm{s}^{-1} \mathrm{~m}^{-3} \mathrm{~nm}^{-1}$ ) at each wavelength is computed from the LVREA and the energy of a photon, which varies with wavelength, as follows:

$$
\left.L V R P A_{\lambda}\right|_{i}=\left[\left.L V R E A_{\lambda}\right|_{i}\right][\lambda /(h c)]
$$

where $h=6.626 \times 10^{-34} \mathrm{Js}$ is the Planck's constant, $c=2.998 \times 10^{8} \mathrm{~m} \mathrm{~s}^{-1}$ is the speed of light, and the bracketed term $[\lambda /(h c)]$ is the energy per photon with wavelength $\lambda$. Since the light source irradiating the photocatalytic system is polychromatic, the total incident irradiance $G$ (e.g., in $\mathrm{J} \mathrm{s}^{-1} \mathrm{~m}^{-2}$ ) and LVRPA (e.g., in photons $\mathrm{s}^{-1} \mathrm{~m}^{-3}$ ) at each segment are obtained by integrating $G_{\lambda}$ and $L V R P A_{\lambda}$, respectively, over the considered wavelength range $\lambda_{1}-\lambda_{2}$ : 


$$
\begin{gathered}
\left.G\right|_{i}=\left.\int_{\lambda_{1}}^{\lambda_{2}} G_{\lambda}\right|_{i} d \lambda \\
\left.L V R P A\right|_{i}=\left.\int_{\lambda_{1}}^{\lambda_{2}} L V R P A_{\lambda}\right|_{i} d \lambda .
\end{gathered}
$$

The wavelength range considered was $360-500 \mathrm{~nm}$, in which the absorption band of the catalyst lies [6]. These calculations were repeated for all segments in the reactor to determine the intensity and LVRPA at each point.

\section{INTRINSIC KINETIC MODEL OF HYDROGEN PRODUCTION USING CADMIUM ZINC SULFIDE CATALYST WITH SULFIDE AND SULFITE}

TABLE II: REACTION MECHANISM FOR HYDROGEN PRODUCTION FROM WATER WITH SULFIDE AND SULFITE IONS [8], [12]

Absorption of photon and electron-hole pair generation

$$
\mathrm{CdZnS}+\mathrm{hv} \rightarrow \mathrm{CdZnS}+\mathrm{e}^{-}+\mathrm{h}^{+}
$$

Recombination of electron and hole

$$
\mathrm{e}^{-}+\mathrm{h}^{+} \rightarrow \phi
$$

Adsorption of reactants

$$
\begin{aligned}
\mathrm{CdZnS}+\mathrm{H}_{2} \mathrm{O} & \rightarrow \mathrm{CdZnS}-\mathrm{H}_{2} \mathrm{O} \\
\mathrm{S}^{2-}+\mathrm{H}_{2} \mathrm{O} & \rightarrow \mathrm{HS}^{-}+\mathrm{OH}^{-} \\
\mathrm{SCdZn}+\mathrm{HS}^{-} & \rightarrow \mathrm{SCdZn}-\mathrm{HS}^{-} \\
\mathrm{SCdZn}+\mathrm{SO}_{3}^{2-} & \rightarrow \mathrm{SCdZn}-\mathrm{SO}_{3}^{2-}
\end{aligned}
$$

$$
\begin{aligned}
& \text { Reactions on the catalyst surface } \\
& \text { Reduction of water to hydrogen } \\
& \mathrm{CdZnS}-\mathrm{H}_{2} \mathrm{O}+\mathrm{SCdZn}+\mathrm{e}^{-} \rightarrow \mathrm{CdZnS}-\mathrm{H}+\mathrm{SCdZn}-\mathrm{OH}^{-} \\
& \quad 2 \mathrm{CdZnS}-\mathrm{H} \bullet \rightarrow 2 \mathrm{CdZnS}+\mathrm{H}_{2} \\
& \text { Oxidation of sulfide } \\
& \mathrm{SCdZn}-\mathrm{HS}^{-}+\mathrm{h}^{+} \rightarrow \mathrm{SCdZn}-\mathrm{HS} \bullet \\
& \mathrm{SCdZn}-\mathrm{HS} \bullet+\mathrm{SCdZn}-\mathrm{OH}^{-} \rightarrow \mathrm{CdZnS}+\mathrm{SCdZn}-\mathrm{S} \bullet^{-}+\mathrm{H}_{2} \mathrm{O} \\
& \mathrm{SCdZn}-\mathrm{HS} \bullet+\mathrm{SCdZn}-\mathrm{S} \bullet-\mathrm{SCdZn}-\mathrm{HS}_{2}^{-}+\mathrm{CdZnS}
\end{aligned}
$$

Desorption of products

$$
\mathrm{SCdZn}-\mathrm{HS}_{2}^{-}+\mathrm{CdZnS}+\mathrm{HS}_{2}^{-}
$$

Reaction of disulfide with sulfite in the liquid phase

$$
\mathrm{HS}_{2}^{-}+\mathrm{SO}_{3}^{2-} \rightarrow \mathrm{S}_{2} \mathrm{O}_{3}^{2-}+\mathrm{HS}^{-}
$$

Adsorption of other species in the system

$$
\begin{aligned}
S C d Z n+\mathrm{OH}^{-} & \rightarrow S C d Z n-O H^{-} \\
S C d Z n+S_{3}^{2-} & \rightarrow S C d Z n-S O_{3}^{2-}
\end{aligned}
$$

\section{A. Kinetic Model}

The photocatalytic hydrogen production on semiconductor particles involves the absorption of photons with energy higher than the catalyst band gap, generating electron-hole pairs, and the reduction by electrons and oxidation by holes of water and sacrificial species, respectively, on the surface of the photocatalyst. A competing step is the recombination of electrons and holes. Table II shows the reaction mechanism for hydrogen production on a cadmium zinc sulfide catalyst, which is assumed to be the same as that postulated for cadmium sulfide catalysts in [8], [12]. The reactions are assumed to take place among adsorbed species on the photocatalyst, following the Langmuir-Hinshelwood mechanism. For brevity, the $\mathrm{Cd}_{\mathrm{x}} \mathrm{Zn}_{1-\mathrm{x}} \mathrm{S}$ photocatalyst site is represented as $\mathrm{CdZnS}$. The symbol CdZnS is used to denote the site on the negatively charged $S$ atom while SCdZn for the site on the positively charged $\mathrm{Cd}$ and $\mathrm{Zn}$ atoms.

The expression for the rate of hydrogen production is derived from the mechanism with the following assumptions: (i) reactions take place among species adsorbed on the catalyst surface, (ii) reactions are elementary and irreversible, (iii) concentrations of electrons, holes, and radicals are at steady-state, (iv) species adsorbed on the photocatalyst are in equilibrium with those in the bulk solution, (v) sulfide, sulfite, and hydroxide ions compete for the same adsorption site, (vi) concentration of water on the catalyst surface is constant, and (vii) the rate of electron-hole generation is proportional to $\gamma^{a, v}$, the LVRPA or the rate of photon absorption per unit volume of catalyst suspension, by the average quantum efficiency $\phi[5]$. A modification to the rate expression was introduced such that the rate retains its dependence on species concentrations when it is reduced to a form having linear dependence with the LVRPA at low intensities. The resulting kinetic rate expression is as follows:

$$
\begin{aligned}
r_{\mathrm{H}_{2}}{ }^{v}= & k\left\{\mathrm{~K}_{\mathrm{HS}^{-}}\left[\mathrm{HS}^{-}\right] /\left(1+\mathrm{K}_{\mathrm{HS}^{-}}\left[\mathrm{HS}^{-}\right]\right.\right. \\
& \left.\left.+\mathrm{K}_{\mathrm{SO}_{3}{ }^{2-}}\left[\mathrm{SO}_{3}{ }^{2-}\right]+\mathrm{K}_{\mathrm{OH}^{-}}\left[\mathrm{OH}^{-}\right]\right)^{2}\right\} \gamma^{a, v}
\end{aligned}
$$

where $r_{H_{2}}^{v}$ is the rate of hydrogen production per unit volume of the suspension, $\mathrm{K}_{\mathrm{HS}^{-}}, \mathrm{K}_{\mathrm{SO}_{3}^{2-}}$, and $\mathrm{K}_{\mathrm{OH}^{-}}$are the adsorption equilibrium constants for $\mathrm{HS}^{-}, \mathrm{SO}_{3}^{2-}$, and $\mathrm{OH}^{-}$, respectively, and the parameter $k$ is:

$$
k=k_{\text {red }}\left[\mathrm{H}_{2} \mathrm{O}\right] k_{\text {ox }}[\mathrm{SCdZn}]_{\text {tot }}{ }^{2} \phi
$$

where $k_{\text {red }}$ is the rate constant for reduction of water to hydrogen, $k_{o x}$ is the rate constant for oxidation of sulfide, $\left[\mathrm{H}_{2} \mathrm{O}\right]$ is the concentration of water on the catalyst surface, and $[\mathrm{SCdZn}]_{\text {tot }}$ represents the total amount of SCdZn sites.

\section{B. Estimation of Kinetic Parameters}

Using the experimental parameters and LVRPA profiles, the model is fit to the experimental rates to estimate the model parameters. By assuming negligible mass transfer limitations since the catalyst particles are kept in suspension by magnetic stirring of the batch reactor contents and that the amount of hydrogen dissolved in the suspension is negligible, the experimentally measured rates of hydrogen production are also taken to be the experimentally determined intrinsic rates of production of this gas.

The experimentally determined rates of hydrogen production presented in Fig. 1 to Fig. 4 are for the entire reactor volume. In order to relate these rates to (11), the expression for $r_{\mathrm{H}_{2}}^{v}$ in (11) must be integrated over the reactor volume $V$ to get the total rate of hydrogen production $r_{\mathrm{H}_{2}}$ in moles of hydrogen per unit time.

$$
\begin{array}{rl}
r_{\mathrm{H}_{2}}=\int_{V} & k\left\{\mathrm{~K}_{\mathrm{HS}^{-}}\left[\mathrm{HS}^{-}\right] /\left(1+\mathrm{K}_{\mathrm{HS}^{-}}\left[\mathrm{HS}^{-}\right]\right.\right. \\
& \left.\left.+\mathrm{K}_{\mathrm{SO}_{3}{ }^{2-}}\left[\mathrm{SO}_{3}{ }^{2-}\right]+\mathrm{K}_{\mathrm{OH}^{-}}\left[\mathrm{OH}^{-}\right]\right)^{2}\right\} \gamma^{a, v} d V
\end{array}
$$


Since the suspension was constantly kept under stirring, the concentration of sulfide, sulfite, and hydroxide ions is assumed to be uniform throughout the reactor. The LVRPA, however, varies with reactor position and must remain in the integral term. Equation (13) may thus be written as:

$$
\begin{aligned}
r_{H_{2}}=k & \left\{\mathrm{~K}_{\mathrm{HS}^{-}}\left[\mathrm{HS}^{-}\right] /\left(1+\mathrm{K}_{\mathrm{HS}^{-}}\left[\mathrm{HS}^{-}\right]\right.\right. \\
& \left.\left.+\mathrm{K}_{\mathrm{SO}_{3}{ }^{2-}}\left[\mathrm{SO}_{3}{ }^{2-}\right]+\mathrm{K}_{\mathrm{OH}^{-}}\left[\mathrm{OH}^{-}\right]\right)^{2}\right\} \int_{V} \gamma^{a, v} d V
\end{aligned}
$$

The reactor cross-sectional area was divided into several segments with length $\Delta r$ and angular width $\Delta \theta$ for the LVRPA profiling. Each of these segment areas is multiplied by the reactor height to obtain the volume $\Delta V$ of each of the differential reactor element for which the LVRPA has been determined. The integration term in (14) is thus carried out numerically as follows.

$$
\begin{aligned}
r_{H_{2}}=k & \left\{\mathrm{~K}_{\mathrm{HS}^{-}}\left[\mathrm{HS}^{-}\right] /\left(1+\mathrm{K}_{\mathrm{HS}^{-}}\left[\mathrm{HS}^{-}\right]\right.\right. \\
& \left.\left.+\mathrm{K}_{\mathrm{SO}_{3}{ }^{2-}}\left[\mathrm{SO}_{3}{ }^{2-}\right]+\mathrm{K}_{\mathrm{OH}^{-}}\left[\mathrm{OH}^{-}\right]\right)^{2}\right\} \sum \gamma^{a, v} \Delta V
\end{aligned}
$$

The kinetic parameter $k$ and the adsorption equilibrium constants $K_{i}$ of species $i$ were estimated by fitting the rate expression given in (15) to the experimental rates (micromol $\mathrm{H}_{2} \mathrm{~h}^{-1}$ ) at the corresponding $\mathrm{HS}^{-}$concentrations (taken to be equal to $\mathrm{Na}_{2} \mathrm{~S}$ concentrations, $\mathrm{M}$ ), $\mathrm{SO}_{3}^{2-}$ concentrations (M), and $\mathrm{OH}^{-}$concentrations (M) given in Table I, and LVRPA (micromol photons $\mathrm{h}^{-1} \mathrm{~cm}^{-3}$ ) profiles. A Marquardt-Levenberg non-linear regression algorithm was used. The resulting values of the parameters are shown in Table III. These parameters are for the temperature of $25^{\circ} \mathrm{C}$, the temperature at which the experimental photocatalytic reaction rates were obtained.

TABLE III: ESTIMATED MODEL PARAMETERS FOR (11) AT $25^{\circ} \mathrm{C}$

$\begin{array}{cc}k & 118.27 \\ K_{\mathrm{HS}^{-}}, \mathrm{L} / \mathrm{mol} & 9,208.58 \\ K_{\mathrm{SO}_{3}^{2-}}, \mathrm{L} / \mathrm{mol} & 7,618.42 \\ K_{\mathrm{OH}^{-}}, \mathrm{L} / \mathrm{mol} & 621.03\end{array}$

Coefficient of determination, $r^{2}=0.8873$

Correlation coefficient, $r=0.9401$

The coefficient of determination and correlation coefficient values indicate good fit of the model to the experimental data. Fig. 6 to Fig. 9 show the parity plots of predicted rates using (15) against the experimental rates for each operating parameter variation. Most of the points pertaining to the predicted rate scatter along the predicted rate $=$ experimental rate line. The relatively large deviations of some points are attributed to radiation field model limitations and to reaction mechanisms that were not taken into account in the kinetic modeling.

Error is relatively large at $0.2 \mathrm{~g} / 100 \mathrm{~mL}$ catalyst loading, as shown in Fig. 6. This may be attributed to the limitations of the radiation field model used due to its simplifications, particularly in the adoption of an isotropic phase function, such that the deviation of the actual distribution of scattering from the assumed form may have become significant at this high catalyst concentration.

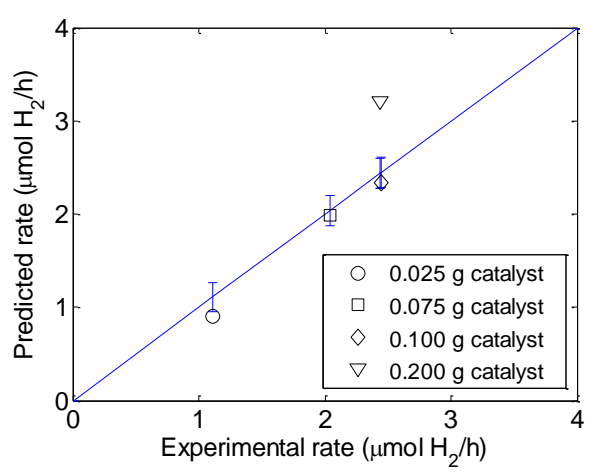

Fig. 6. Plot of predicted rate vs. experimental rate of hydrogen production at different catalyst amounts (in $100 \mathrm{~mL}$ solution with $0.1 \mathrm{M} \mathrm{Na}_{2} \mathrm{~S}, 0.1 \mathrm{M}$ $\mathrm{Na}_{2} \mathrm{SO}_{3}, 54.95 \mathrm{~mW} / \mathrm{cm}^{2}$ incident irradiance).

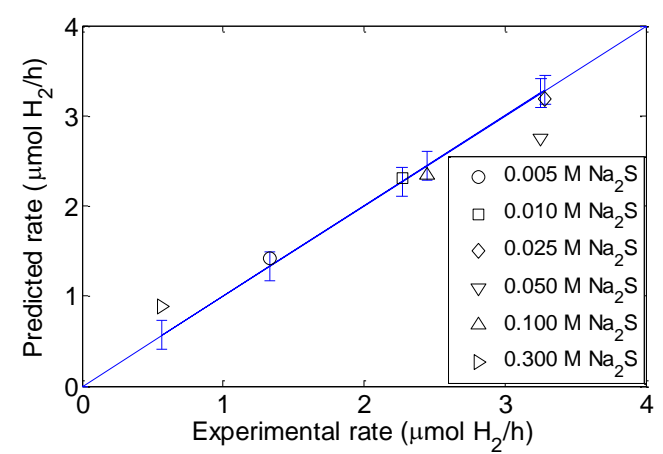

Fig. 7. Plot of predicted rate vs. experimental rate of hydrogen production at different $\mathrm{Na}_{2} \mathrm{~S}$ concentrations $(0.1 \mathrm{~g}$ catalyst in $100 \mathrm{~mL}$ solution with $0.1 \mathrm{M}$ $\mathrm{Na}_{2} \mathrm{SO}_{3}, 54.95 \mathrm{~mW} / \mathrm{cm}^{2}$ incident irradiance).

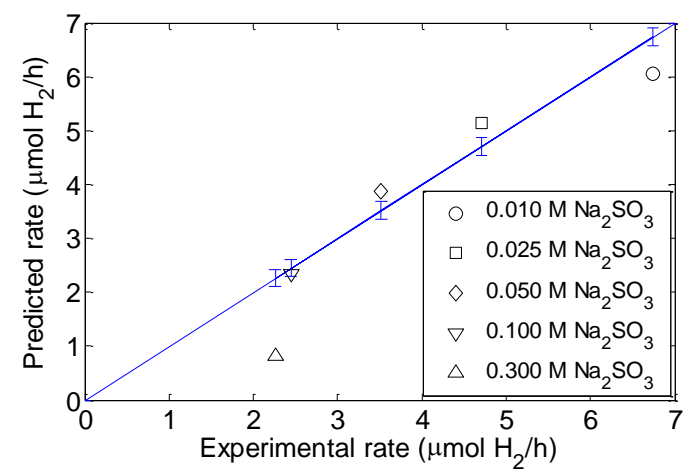

Fig. 8. Plot of predicted rate vs. experimental rate of hydrogen production at different $\mathrm{Na}_{2} \mathrm{SO}_{3}$ concentrations $(0.1 \mathrm{~g}$ catalyst in $100 \mathrm{~mL}$ solution with 0.1 $\mathrm{M} \mathrm{Na}_{2} \mathrm{~S}, 54.95 \mathrm{~mW} / \mathrm{cm}^{2}$ incident irradiance).

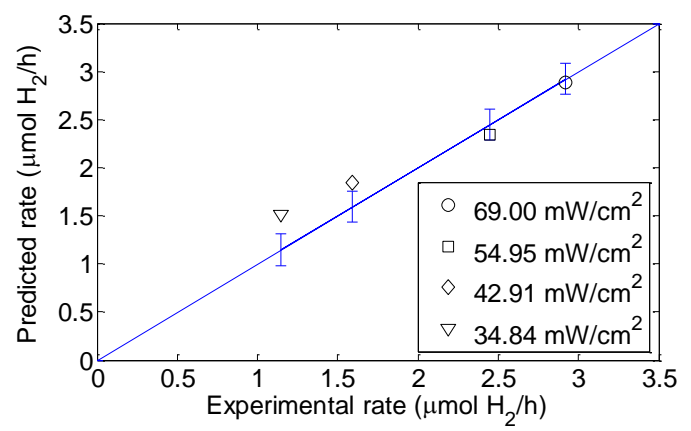

Fig. 9. Plot of predicted rate vs. experimental rate of hydrogen production at different incident irradiances $(0.1 \mathrm{~g}$ catalyst in $100 \mathrm{~mL}$ solution with $0.1 \mathrm{M}$ $\mathrm{Na}_{2} \mathrm{~S}, 0.1 \mathrm{M} \mathrm{Na}_{2} \mathrm{SO}_{3}$ ).

At varied sulfite concentrations shown in Fig. 8, error is relatively large at $0.300 \mathrm{M}$. The higher rate of hydrogen production than that predicted may be attributed to the participation of sulfite in the consumption of holes when 
present in high amounts in the solution as indicated in the following reaction, which was not included in the kinetic modeling.

$$
\mathrm{SO}_{3}{ }^{2-}+2 \mathrm{OH}^{-}+2 \mathrm{~h}^{+} \rightarrow \mathrm{SO}_{4}{ }^{2-}+\mathrm{H}_{2} \mathrm{O}
$$

In addition to the consumption of holes by sulfide ions, oxidation of sulfite ions by the photogenerated holes further prevents electron-hole recombination, increasing the rate of hydrogen production.

\section{Limitations of the Model}

The developed kinetic model considers only the species initially present in the system and does not include the influence of products such as thiosulfates and disulfides on the rate as they are formed. Only the photo-oxidation of sulfide is considered in the mechanism; the participation of sulfite in reactions with photogenerated holes at high sulfite concentrations is not taken into account.

Since the model parameters were estimated using the LVRPA values derived from the model of the radiation field in the reactor, the parameters may be further refined by applying more rigorous radiation modeling procedures employing an anistropic phase function. The resulting model in this work is only valid for catalyst concentrations less than the optimum, which could be attributed to the adoption of an isotropic phase function in the determination of the LVRPA.

The model is limited to the linear-dependence of the rate of hydrogen production on the LVRPA. This linear-dependence regime has also been observed in other water splitting systems irradiated at intensities in the same order of magnitude as solar radiation in the visible light region. This model may not be able to predict the rate of hydrogen production at high intensities in the UV region, at which studies have shown the rate of hydrogen production on UV-active photocatalysts to have a sub-linear dependence on the LVRPA.

\section{CONCLUSION AND RECOMMENDATIONS}

The rate of hydrogen production on a $\mathrm{Cd}_{0.4} \mathrm{Zn}_{0.6} \mathrm{~S}$ catalyst was investigated at varying catalyst amounts, $\mathrm{Na}_{2} \mathrm{~S}$ concentrations, $\mathrm{Na}_{2} \mathrm{SO}_{3}$ concentrations, and incident light intensities. The dependence of the hydrogen generation rate on these parameters was explained by their effect on the rate of electron-hole generation, suppression of electron-hole recombination, irradiance profile, and competitive adsorption on catalyst sites. The intensity and LVRPA profiles in the photoreactor were determined using an improved procedure that applies the theory of Kubelka and Munk.

An intrinsic kinetic model reflecting the influence of the sulfide, sulfite, and hydroxide (from the hydrolysis of the sacrificial reagents) concentrations and the LVRPA was developed. The model shows Langmuir-Hinshelwood characteristics in the concentration-dependent term and linear dependence of the rate on the LVRPA valid at low intensities. The model, with four parameters - a kinetic parameter and the adsorption equilibrium constants of sulfide, sulfite, and hydroxide ions - estimated from experimental data, shows good fit to the experimental data. Since the experimental conditions such as the irradiation form, reactor geometry, and lamp-reactor configuration were defined and taken into account in the determination of the LVRPA, the estimated model parameters may be used to provide information for the photoreaction kinetics component in photocatalytic reactor design and scale-up using the $\mathrm{Cd}_{0.4} \mathrm{Zn}_{0.6} \mathrm{~S}$ catalyst for the photocatalytic production of hydrogen.

Validation of the computed absorption and scattering coefficients of the cadmium zinc sulfide particles in water and the developed intrinsic kinetic model to other photoreactor configurations is recommended. The optical properties and the model parameters may be further improved by solving the complete form of the RTE applied to the irradiated photocatalyst suspensions. The model may also be extended to take into account the influence of the products such as thiosulfate and the participation of sulfite in the oxidation reactions at high sulfite concentrations.

\section{ACKNOWLEDGMENT}

The authors thank the Department of Chemical Engineering Laboratory, the Department of Mechanical Engineering Machine Shop, the College of Engineering Building Maintenance Office, and the Photonics Research Laboratory of the National Institute of Physics of the University of the Philippines Diliman for their assistance in the experimental parts of this work.

\section{REFERENCES}

[1] M. R. Pai, A. M. Banerjee, A. K. Tripathi, and S. R. Bharadwaj, "Fundamentals and Applications of the Photocatalytic Water Splitting Reaction," in Functional Materials: Preparation, Processing and Applications, S. R. Banerjee and A. K. Tyagi, Eds. London: Elsevier, 2012, pp. 579-581.

[2] X. Chen, S. Shen, L. Guo, and S. S. Mao, "Semiconductor-based Photocatalytic Hydrogen Generation," Chemical Reviews, vol. 110, no. 11, pp. 6503-6570, May 2010.

[3] C. Xing, Y. Zhang, W. Yan, and L. Guo, "Band structure-controlled solid solution of $\mathrm{Cd}_{1-x} \mathrm{Zn}_{x} \mathrm{~S}$ photocatalyst for hydrogen production by water splitting," International Journal of Hydrogen Energy, vol. 31, pp. 2018-2024, March 2006

[4] D. Jing, et al., "Efficient solar hydrogen production by photocatalytic water splitting: From fundamental study to pilot demonstration,' International Journal of Hydrogen Energy, vol. 35, pp. 7087-7097, January 2010

[5] J. Marugán, R. van Grieken, A. E. Cassano, and O. M. Alfano, "Intrinsic Kinetic Modeling with Explicit Radiation Absorption Effects of the Photocatalytic Oxidation of Cyanide with $\mathrm{TiO}_{2}$ and Silica-Supported $\mathrm{TiO}_{2}$ Suspensions," Applied Catalysis B. Environmental, vol. 85, pp. 48-60, 2008.

[6] E. del Rosario, L. Doon, P. N. Perez, R. de Leon, and H. Ramos, "Hydrogen Production by Visible Light-driven Photocatalytic Water Splitting using Plasma-enhanced $\mathrm{CdZn}_{1-\mathrm{x}} \mathrm{S}$," unpublished manuscript, University of the Philippines Diliman, PH.

[7] R. Priya, "Solar Photocatalytic Generation of Hydrogen From Hydrogen Sulfide in an Alkaline Solution using $\mathrm{CdS}-\mathrm{ZnS} / \mathrm{TiO}_{2}$ Nanocomposites." Ph.D. dissertation, Centre for Environmental Studies, Anna University, Chenai, 2011.

[8] J. Sabate, S. Cervera-March, R. Simarro, and J. Gimenez, "Photocatalytic production of hydrogen from sulfide and sulfite waste streams: A kinetic model for reactions occurring in illuminated suspensions of CdS," Chemical Engineering Science, vol. 45, pp. 3089-3096, January 1990.

[9] M. N. Ozisik, Radiative Transfer and Interactions with Conduction and Convection, New York: Wiley, 1973.

[10] G. Kortum, Reflectance Spectroscopy: Principles, Methods Applications, New York: Springer, 1969, pp. 103-127.

[11] G. Palmisano, V. Loddo, and V. Augugliaro. (June 2013) Two-Dimensional Modeling of an Externally Irradiated Slurry Photoreactor. International Journal of Chemical Reactor Engineering. [Online]. 11(2). pp. 675-685. Available: 
http://www.degruyter.com/dg/viewarticle/j\$002fijcre.2013.11.issue-2 \$002fijcre-2012-0049\$002fijcre-2012-0049.xml

[12] S. Y. Ryu, J. Choi, W. Balcerski, T. K. Lee, and M. R. Hoffmann "Photocatalytic Production of $\mathrm{H}_{2}$ on Nanocomposite Catalysts," Industrial \& Engineering Chemistry Research, vol. 46, no. 23, pp, 7467-7488, June 2007.

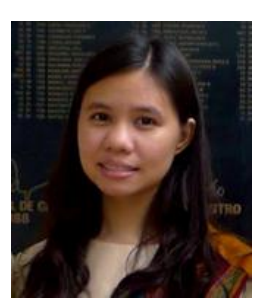

Hyacinth Mae G. Tambago earned her bachelor's degree and master's degree in chemical engineering from the University of the Philippines, Diliman, Quezon City, Philippines.

She has been an instructor of the Department of Chemical Engineering at the University of the Philippines Diliman since 2010. Her research interests are photocatalytic hydrogen production and photocatalytic reaction engineering.

Ms. Tambago is a member of the Philippine Institute of Chemical
Engineers - Metro Manila Academe Chapter.

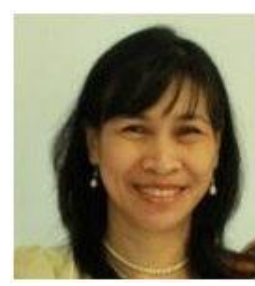

Rizalinda L. de Leon earned her bachelor's degree in chemical engineering, her master's degree in energy engineering, and her $\mathrm{PhD}$ degree in chemical engineering at the University of the Philippines, Diliman, Quezon City.

She is currently an assistant professor of chemical engineering at the University of the Philippines Diliman. Her research interests include photocatalytic hydrogen production, nanofluids, and bioethanol production.

Dr. de Leon is a member of the Philippine Institute of Chemical Engineers. 